\title{
Theory of 3-D angle gathers in wave-equation seismic imaging
}

\author{
Sergey Fomel
}

Received: 26 September 2010/ Accepted: 24 January 2011/Published online: 22 February 2011

(c) The Author(s) 2011. This article is published with open access at Springerlink.com

\begin{abstract}
I present two methods for constructing angle gathers in 3-D seismic imaging by downward extrapolation. Angles in angle gathers refer to the scattering angle at the reflector and provide a natural access to analyzing migration velocity and amplitudes. In the first method, angle gathers are extracted at each downward-continuation step by mapping transformations in constant-depth frequency slices. In the second method, one extracts angle gathers after applying the imaging condition by transforming local offset gathers in the depth domain. The second approach generalizes previously published algorithms for angle-gather construction in 2-D and commonazimuth imaging.
\end{abstract}

Keywords Geophysics · Seismic imaging ·

Velocity analysis · Amplitude analysis

\section{Introduction}

Wave extrapolation provides an accurate method for seismic imaging in structurally complex areas (Biondi 2006; Etgen et al. 2009). Wave extrapolation methods have several known advantages in comparison with direct methods such as Kirchhoff migration thanks to their ability to handle multi-pathing, strong velocity heterogeneities, and finite-bandwidth wave-propagation effects (Gray et al. 2001). However, velocity and amplitude analysis in the prestack domain are not immediately available for wave

\section{S. Fomel $(\square)$}

Bureau of Economic Geology, John A. and Katherine G. Jackson School of Geosciences, The University of Texas at Austin, University Station, Box X, Austin, TX 78713-8924, USA e-mail: sergey.fomel@beg.utexas.edu extrapolation methods. To overcome this limitation, several authors (de Bruin et al. 1990; Prucha et al. 1999; Mosher and Foster 2000; Rickett and Sava 2002; Xie and Wu 2002; Soubaras 2003; Sava and Fomel 2003, 2005, 2006) suggested methods for constructing angle gathers from downward-continued wavefields. Angles in angle gathers are generally understood as the reflection (scattering) angles at reflecting interfaces (Xu et al. 2001; Brandsberg-Dahl et al. 2003). Angle gathers facilitate velocity analysis (Liu et al. 2001; Stork et al. 2002) and can be used in principle for extracting angle-dependent reflectivity information directly at the target reflectors (Sava et al. 2001). Stolk and de Hoop (2002) assert that angle gathers generated with wavefield extrapolation are genuinely free of artifacts documented for Kirchhoff-generated angle gathers (Stolk and Symes 2002, 2004).

There are two possible approaches to angle-gather construction with wavefield continuation. In the first approach, one generates gathers at each depth level converting offset-space-frequency planes into angle-space planes simultaneously with applying the imaging condition. The offset in this case refers to the local offset between source and receiver parts of the downward continued prestack data. Such a construction was suggested, for example, by Prucha et al. (1999). This approach is attractive because of its localization in depth. However, the method of Prucha et al. (1999) produces gathers in the offset ray parameter as opposed to angle. As a result, the angle-domain information becomes structure-dependent: the output depends not only on the scattering angle but also on the structural dip.

In the second approach, one converts migrated images in offset-depth domain to angle-depth gathers after imaging of all depth levels is completed. Sava and Fomel (2003) suggested a simple Radon-transform procedure for 
extracting angle gathers from migrated images. The transformation is independent of velocity and structure. Rickett and Sava (2002) adopted it for constructing angle gathers in the shot-gather migration. Biondi and Symes (2004) demonstrate that the method of Sava and Fomel (2003) is strictly valid in the 3-D case only in the absence of cross-line structural dips. They present an extension of this method for the common-azimuth approximation (Biondi and Palacharla 1996).

In this paper, I present a more complete analysis of the angle-gather construction in 3-D imaging by wavefield continuation. First, I show how to remove the structural dependence in the depth-slice approach. The improved mapping retains the velocity dependence but removes the effect of the structure. Additionally, I extend the second, post-migration approach to a complete 3-D wide-azimuth situation. Under the common-azimuth approximation, this formulation reduces to the result of Biondi et al. (2003) and, in the absence of cross-line structure, it is equivalent to the Radon construction of Sava and Fomel (2003).

\section{Traveltime derivatives and dispersion relationships for a 3-D dipping reflector}

Theoretical analysis of angle gathers in downward continuation methods can be reduced to analyzing the geometry of reflection in the simple case of a dipping reflector in a locally homogeneous medium. Considering the reflection geometry in the case of a plane reflector is sufficient for deriving relationships for local reflection travel time derivatives in the vicinity of a reflection point (Goldin 2002). Let the local reflection plane be described in $\{x, y, z\}$ coordinates by the general equation

$x \cos \alpha+y \cos \beta+z \cos \gamma=d$,

where the normal angles $\alpha, \beta$, and $\gamma$ satisfy

$\cos ^{2} \alpha+\cos ^{2} \beta+\cos ^{2} \gamma=1$,

The geometry of the reflection ray paths is depicted in Fig. 1. The reflection travel time measured on a horizontal surface above the reflector is given by the known expression (Slotnick 1959; Levin 1971)

$t\left(h_{x}, h_{y}\right)=\frac{2}{v} \sqrt{D^{2}+h_{x}^{2}+h_{y}^{2}-\left(h_{x} \cos \alpha+h_{y} \cos \beta\right)^{2}}$,

where $D$ is the length of the normal to the reflector from the midpoint (distance $M M \prime$ in Fig. 2)

$D=d-m_{x} \cos \alpha-m_{y} \cos \beta$,

$m_{x}$ and $m_{y}$ are the midpoint coordinates, $h_{x}$ and $h_{y}$ are the half-offset coordinates, and $v$ is the local propagation velocity.

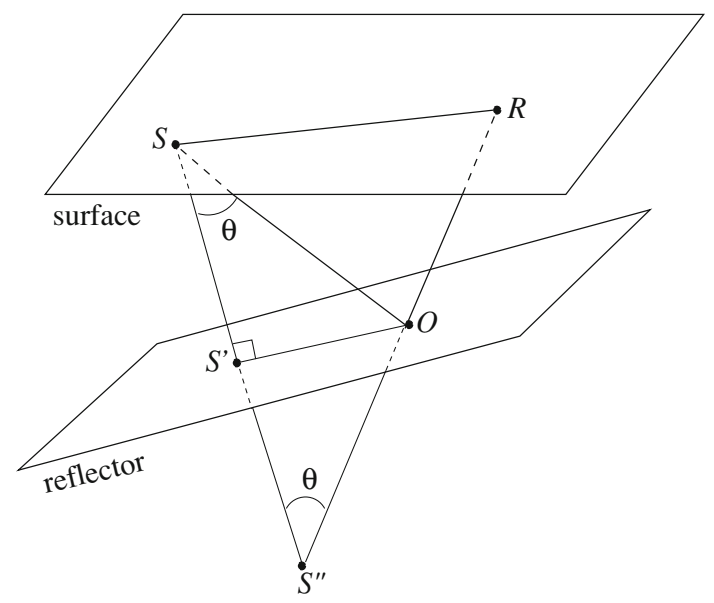

Fig. 1 Reflection geometry in 3-D (a scheme). $S$ and $R$ and the source and the receiver positions at the surface. $O$ is the reflection point. $S /$ is the normal projection of the source to the reflector. $S / \prime$ is the "mirror" source. The cumulative length of the incident and reflected rays is equal to the distance from $S / \prime$ to $R$

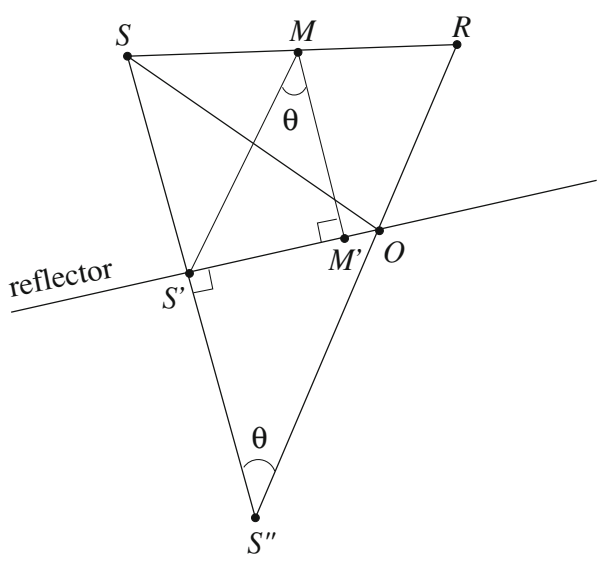

Fig. 2 Reflection geometry in the reflection plane (a scheme). $M$ is the midpoint. As follows from the similarity of triangles $S / I S R$ and $S / S M$, the distance from $M$ to $S /$ is twice smaller than the distance from $S / l$ to $R$

According to elementary geometrical considerations (Figs. 1, 2), the reflection angle $\theta$ is related to the previously introduced quantities by the equation

$\cos \theta=\frac{D}{\sqrt{D^{2}+h_{x}^{2}+h_{y}^{2}-\left(h_{x} \cos \alpha+h_{y} \cos \beta\right)^{2}}}$.

Explicitly differentiating Eq. 3 with respect to the midpoint and offset coordinates and utilizing Eq. 5 leads to the equations

$t_{m_{x}} \equiv \frac{\partial t}{\partial m_{x}}=-\frac{2}{v} \cos \theta \cos \alpha$

$t_{m_{y}} \equiv \frac{\partial t}{\partial m_{y}}=-\frac{2}{v} \cos \theta \cos \beta$, 
$t_{h_{x}} \equiv \frac{\partial t}{\partial h_{x}}=\frac{4}{v^{2} t}\left(h_{x} \sin ^{2} \alpha-h_{y} \cos \alpha \cos \beta\right)$,

$t_{h_{y}} \equiv \frac{\partial t}{\partial h_{y}}=\frac{4}{v^{2} t}\left(h_{y} \sin ^{2} \beta-h_{x} \cos \alpha \cos \beta\right)$.

Additionally, the traveltime derivative with respect to the depth of the observation surface is given by

$t_{z} \equiv \frac{\partial t}{\partial z}=-\frac{2}{v} \cos \theta \cos \gamma$

and is related to the previously defined derivatives by the double-square-root equation

$$
\begin{aligned}
-v t_{z}= & \sqrt{1-\frac{v^{2}}{4}\left(t_{m_{x}}-t_{h_{x}}\right)^{2}-\frac{v^{2}}{4}\left(t_{m_{y}}-t_{h_{y}}\right)^{2}} \\
& +\sqrt{1-\frac{v^{2}}{4}\left(t_{m_{x}}+t_{h_{x}}\right)^{2}-\frac{v^{2}}{4}\left(t_{m_{y}}+t_{h_{y}}\right)^{2}} .
\end{aligned}
$$

In the frequency-wavenumber domain, Eq. 11 serves as the basis for 3-D shot-geophone downward-continuation imaging. In the Fourier domain, each $t_{x}$ derivative translates into $-k_{x} / \omega$ ratio, where $k_{x}$ is the wavenumber corresponding to $x$ and $\omega$ is the temporal frequency.

Equations (6), (7), and (10) immediately produce the first important 3-D relationship for angle gathers

$\cos \theta=\frac{v}{2 \omega} \sqrt{k_{m_{x}}^{2}+k_{m_{y}}^{2}+k_{z}^{2}}$.

Expressing the depth derivative with the help of the double-square-root Eq. 11 and applying a number of algebraic transformations, one can turn Eq. 12 into the dispersion relationship

$$
\begin{array}{r}
\left(k_{m_{x}}^{2}+k_{m_{y}}^{2}\right) \frac{\sin ^{2} \theta}{v^{2}}+\left(k_{h_{x}}^{2}+k_{h_{y}}^{2}\right) \frac{\cos ^{2} \theta}{v^{2}} \\
=\frac{1}{4 \omega^{2}}\left(k_{m_{x}} k_{h_{y}}-k_{m_{y}} k_{h_{x}}\right)^{2}+4 \omega^{2} \frac{\cos ^{2} \theta}{v^{2}} \frac{\sin ^{2} \theta}{v^{2}} .
\end{array}
$$

For each reflection angle $\theta$ and each frequency $\omega$, Eq. 13 specifies the locations on the four-dimensional $\left(k_{m_{x}}, k_{m_{y}}, k_{h_{x}}, k_{h_{y}}\right)$ wavenumber hyperplane that contribute to the common-angle gather. In the 2-D case, Eq. 13 simplifies by setting $k_{h_{y}}$ and $k_{y}$ to zero. Using the notation $k_{m_{x}}=k_{m}$ and $k_{h_{x}}=k_{h}$, the 2-D equation takes the form

$k_{m}^{2} \sin ^{2} \theta+k_{h}^{2} \cos ^{2} \theta=\frac{4 \omega^{2}}{v^{2}} \cos ^{2} \theta \sin ^{2} \theta$

and can be explicitly solved for $k_{h}$ resulting in the convenient 2-D dispersion relationship

$k_{h}=\frac{2 \omega \sin \theta}{v} \sqrt{1-\frac{4 k_{m}^{2} v^{2}}{\omega^{2} \cos ^{2} \theta}}$.

In the next section, I show that a similar simplification is also valid under the common-azimuth approximation. Equations (13) and (15) describe an effective migration of the downward-continued data to the appropriate positions on midpoint-offset planes to remove the structural dependence from the local image gathers.

Another important relationship follows from eliminating the local velocity $v$ from Eqs. 11 and 12 . Expressing $v^{2}$ from Eq. 12 and substituting the result in Eq. 12, we arrive (after a number of algebraical transformations) to the frequency-independent equation

$\tan ^{2} \theta=\frac{k_{z}^{2}\left(k_{h_{x}}^{2}+k_{h_{y}}^{2}\right)+\left(k_{h_{x}} k_{m_{x}}+k_{h_{y}} k_{m_{y}}\right)^{2}}{k_{z}^{2}\left(k_{m_{x}}^{2}+k_{m_{y}}^{2}+k_{z}^{2}\right)}$.

Equation (16) can be expressed in terms of ratios $k_{m_{x}} / k_{z}$ and $k_{m_{y}} / k_{z}$, which correspond at the zero local offset to local structural dips ( $z_{m_{x}}$ and $z_{m_{y}}$ partial derivatives), and ratios $k_{h_{x}} / k_{z}$ and $k_{h_{y}} / k_{z}$, which correspond to local offset slopes. As shown by Sava and Fomel (2005), it can be also expressed as

$\tan ^{2} \theta=\frac{k_{h_{x}}^{2}+k_{h_{y}}^{2}+k_{h_{z}}^{2}}{k_{m_{x}}^{2}+k_{m_{y}}^{2}+k_{z}^{2}}$

where $k_{h_{z}}$ refers to the vertical offset between source and receiver wavefields (Biondi and Shan 2002).

In the 2-D case, Eq. 16 simplifies to the form, independent of the structural dip:

$\tan \theta=\frac{k_{h}}{k_{z}}$,

which is the equation suggested by Sava and Fomel (2003). Equation (18) appeared previously in the theory of migration-inversion (Stolt and Weglein 1985).

\section{Common-azimuth approximation}

Common-azimuth migration (Biondi and Palacharla 1996) is a downward continuation imaging method tailored for narrow-azimuth streamer surveys that can be transformed to a single common azimuth with the help of azimuth moveout (Biondi et al. 1998). Employing the commonazimuth approximation, one assumes the reflection plane stays confined in the acquisition azimuth. Although this assumption is strictly valid only in the case of constant velocity (Vaillant and Biondi 2000), the modest azimuth variation in realistic situations justifies the use of the method (Biondi 2003).

To restrict equations of the previous section to the common-azimuth approximation, it is sufficient to set the cross-line offset $h_{y}$ to zero assuming the $x$ coordinate is oriented along the acquisition azimuth. In particular, from Eqs. 8, 9, we obtain

$h_{x} \sin \alpha=\frac{v t}{2} \sin \theta$ 
$t_{h_{x}}=\frac{4 h_{x}}{v^{2} t} \sin ^{2} \alpha=\frac{2}{v} \sin \theta \sin \alpha$,

$t_{h_{y}}=-\frac{4 h_{x}}{v^{2} t} \cos \alpha \cos \beta=-\frac{2}{v} \sin \theta \cot \alpha \cos \beta$.

With the help of Eqs. 6, 7, and 10), Eq. 21 transforms to the form

$$
\begin{aligned}
t_{h_{y}} & =t_{m_{y}} \frac{\tan \theta}{\tan \alpha} \\
& =t_{m_{y}} \frac{\sqrt{1-\frac{v^{2}}{4}\left(t_{m_{x}}+t_{h_{x}}\right)^{2}}-\sqrt{1-\frac{v^{2}}{4}\left(t_{m_{x}}-t_{h_{x}}\right)^{2}}}{\sqrt{1-\frac{v^{2}}{4}\left(t_{m_{x}}+t_{h_{x}}\right)^{2}}+\sqrt{1-\frac{v^{2}}{4}\left(t_{m_{x}}-t_{h_{x}}\right)^{2}}},
\end{aligned}
$$

suggested by Biondi and Palacharla (1996). Combining Eqs. 6, 7, 10, and 20 and transforming to the frequencywavenumber domain, we obtain the common-azimuth dispersion relationship

$\left(k_{h_{x}}^{2}+k_{m_{y}}^{2}+k_{z}^{2}\right)\left(k_{m_{x}}^{2}+k_{m_{y}}^{2}+k_{z}^{2}\right)=\frac{4 \omega^{2}}{v^{2}}\left(k_{m_{y}}^{2}+k_{z}^{2}\right)$,

which shows that, under the common-azimuth approximation and in a laterally homogeneous medium, 3-D seismic migration amounts to a cascade of a 2-D prestack migrations in the in-line direction and a 2-D zerooffset migration in the cross-line direction (Canning and Gardner 1996).

Under the common-azimuth approximation, the angledependent relationship (13) takes the form

$k_{m_{x}}^{2} \sin ^{2} \theta+k_{h_{x}}^{2} \cos ^{2} \theta=\frac{4 \omega^{2}}{v^{2}} \cos ^{2} \theta \sin ^{2} \theta$

which is identical to the 2-D Eq. 14. This proves that under this approximation, one can perform the structural correction independently for each cross-line wavenumber.

The post-imaging Eq. 16 transforms to the equation

$\tan ^{2} \theta=\frac{k_{h_{x}}^{2}}{k_{m_{y}}^{2}+k_{z}^{2}}$,

obtained previously by Biondi et al. (2003). In the absence of cross-line structural dips $\left(k_{m_{y}}=0\right)$, it is equivalent to the 2-D Eq. 18 .

\section{Algorithm I: Angle gathers during downward continuation}

This algorithm follows from Eq. 13. It consists of the following steps, applied at each propagation depth $z$ :

1. Generate local offset gathers and transform them to the wavenumber domain. In the double-square-root migration, the local offset wavenumbers are immediately available. In the shot gather migration, local offsets are generated by cross-correlation of the source and receiver wavefields (Rickett and Sava 2002).

2. For each frequency $\omega$, transform the local offset wavenumbers $k_{h_{x}}, k_{h_{y}}$ into the angle coordinates $\sin \theta / v$ according to Eq. 13. The angle coordinates depend on velocity but do not depend on the local structural dip. In the 2-D case, each frequency slice is simply the $k_{m}, k_{h}$ plane, and each angle coordinate corresponds to a circle in that plane centered at the origin and described by Eq. 14. Figure 3 shows an example of a 2-D frequency slice transformed to angles.

3. Accumulate contributions from all frequencies to apply the imaging condition in time.

This algorithm is applicable for targets localized in depth. The local offset gathers need to be computed for all lateral locations, but there is no need to store them in memory, because conversion to angles happens on the fly. The algorithm outputs not angles directly, but velocitydependent parameters $\sin \theta / v$. Alkhalifah and Fomel (2009, 2011) have recently extended this algorithm to transversally isotropic media.

\section{Algorithm II: Post-migration angle gathers}

The second algorithm follows from Eq. 16. It applies after the imaging has completed and consists of the following steps applied at each common-image location:

1. Generate and store local offset gathers. In the doublesquare-root migration, the local offsets are immediately available. In the shot gather migration, local

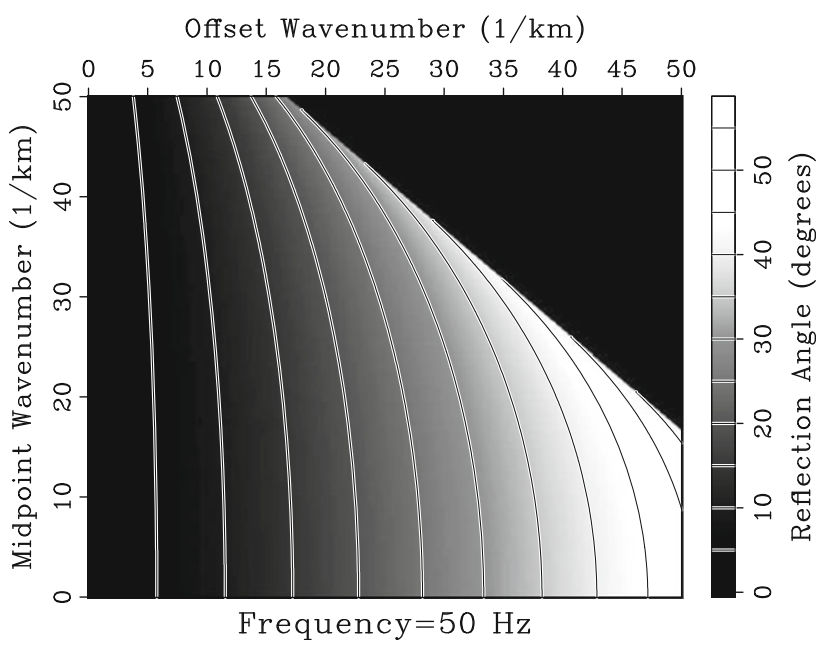

Fig. 3 Constant-depth constant-frequency slice mapped to reflection angles according to the 2-D version of Algorithm I. Zero offset wavenumber maps to zero (normal incidence) angle. The top right corner is the evanescent region 
Fig. 4 Mapping from the offset slope plane to angles according to Algorithm II. Zero slopes map to zero (normal-incidence) angle

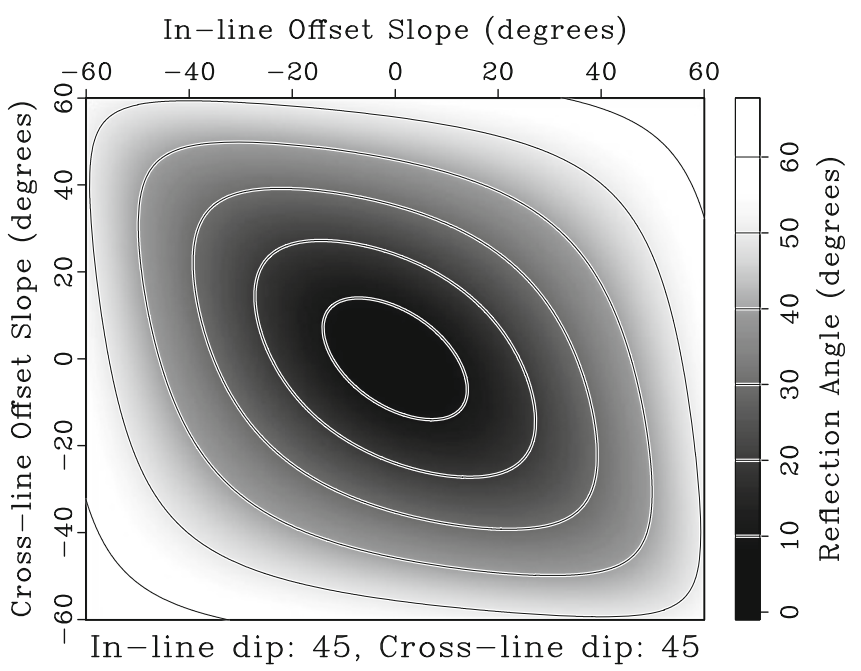

the literature. By the end of 2000s, prestack 3-D reversetime migration has become a standard tool for depth imaging in structurally-complex areas, and it is becoming feasible to generate 3-D angle gathers as part of routine processing (Luo et al. 2010; Vyas et al. 2010; Xu et al. 2010). The most important new theoretical developments are the ability to extract angle information from time-shift angle gathers (Sava and Fomel 2006; Vyas et al. 2010), the ability to extract not only reflection-angle but also azimuth information ( $\mathrm{Xu}$ et al. 2010), and the extension of the angle-gather theory to anisotropy (Biondi 2007; Alkhalifah and Fomel 2009, 2011).

\section{Conclusions}

Angle gathers present a natural tool for analyzing velocities and amplitudes in wave-equation imaging. I have discussed two approaches for angle-gather construction. In the first approach, angle gathers are constructed on the fly at different depth steps of the wave extrapolation process. In the second approach, angle gathers are extracted from the local-offset gathers after imaging has completed. The second method was previously presented for the 2-D case and for the case of a common-azimuth approximation. Both approaches have advantages and disadvantages. The preference depends on the application and the input data configuration.

Acknowledgments I am grateful to Nanxun Dai, John Etgen, Sergey Goldin, and Paul Sava for enlightening discussions.

This publication is authorized by the Director, Bureau of Economic Geology, The University of Texas at Austin.

Open Access This article is distributed under the terms of the Creative Commons Attribution Noncommercial License which permits any noncommercial use, distribution, and reproduction in any medium, provided the original author(s) and source are credited.

(Fomel 2004), many new research results have appeared in 


\section{References}

Alkhalifah T, Fomel S (2009) Angle gathers in wave-equation imaging for VTI media. In: 79th Ann. Internat. Mtg, Soc. Expl. Geophys., Expanded Abstracts. Soc. of Expl. Geophys., pp 2899-2903.

Alkhalifah T, Fomel S (2011) Angle gathers in wave equation imaging for transversely isotropic media. Geophys Prospect, vol 59. doi:10.1111/j.1365-2478.2010.00930.x

Biondi B (2003) Narrow-azimuth migration of marine streamer data. In: 73rd Ann. Internat. Mtg, Soc. Expl. Geophys. Expanded Abstracts. Soc. of Expl. Geophys., pp 897-900

Biondi B (2007) Angle-domain common-image gathers from anisotropic migration. Geophysics 72:581-591

Biondi B, Fomel S, Chemingui N (1998) Azimuth moveout for 3-D prestack imaging. Geophysics 63:574-588

Biondi B, Palacharla G (1996) 3-D prestack migration of commonazimuth data. Geophysics 61:1822-1832

Biondi B, Shan G (2002) Prestack imaging of overturned reflections by reverse time migration. In: 72nd Ann. Internat. Mtg, Soc. Expl. Geophys., pp 1284-1287

Biondi B, Symes W (2004) Angle-domain common-image gathers for migration velocity analysis by wavefield-continuation methods. Geophysics 69:1283-1298

Biondi B, Tisserant T, Symes W (2003) Wavefield-continuation angle-domain common-image gathers for migration velocity analysis. In: 73rd Ann. Internat. Mtg, Soc. Expl. Geophys., Expanded Abstracts. Soc. of Expl. Geophys., pp 2104-2107

Biondi BL (2006) 3-D seismic imaging. Society of Exploration Geophysicists

Brandsberg-Dahl S, de Hoop MV, Ursin B (2003) Focusing in dip and AVA compensation on scattering-angle/azimuth common image gathers. Geophysics 68:232-254

Canning A, GHF Gardner (1996) A two-pass approximation to 3-D prestack migration. Geophysics 61:409-421

de Bruin CGM, Wapenaar CPA, AJ Berkhout (1990) Angledependent reflectivity by means of prestack migration. Geophysics 55:1223-1234

Etgen J, Gray SH, Zhang Y (2009) An overview of depth imaging in exploration geophysics. Geophysics 74:WCA5-WCA17

Fomel S (2004) Theory of 3D angle gathers in wave-equation imaging. In: 74th Ann. Internat. Mtg., Soc. of Expl. Geophys., pp 1053-1056

Goldin SV (2002) Theoretical aspects of 3-D DMO. In: 72nd Ann. Internat. Mtg, Soc. Expl. Geophys., Expanded Abstracts. Soc. of Expl. Geophys., pp 2333-2336.

Gray SH, Etgen J, Dellinger J, Whitmore D (2001) Seismic migration problems and solutions. Geophysics 66:1622-1640

Levin FK (1971) Apparent velocity from dipping interface reflections. Geophysics 36: 510-516 (Errata in GEO-50-11-2279)

Liu W, Popovici A, Bevc D, Biondi B (2001) 3D migration velocity analysis for common image gathers in the reflection angle domain. In: 71st Ann. Internat. Mtg, Soc. of Expl. Geophys., pp $885-888$

Luo M, Lu R, Winbow G, Bear L (2010) A comparison of methods for obtaining local image gathers in depth migration. In: 80th Ann. Internat. Mtg, Soc. Expl. Geophys., Expanded Abstracts. Soc. of Expl. Geophys., pp 3247-3251.
Mosher C, Foster D (2000) Common angle imaging conditions for prestack depth migration. In: 70th Ann. Internat. Mtg, Soc. of Expl. Geophys., pp 830-833.

Prucha M, Biondi B, Symes W (1999) Angle-domain common image gathers by wave-equation migration. In: 69th Ann. Internat. Mtg, Soc. of Expl. Geophys., pp 824-827.

Rickett JE, PC Sava (2002) Offset and angle-domain common imagepoint gathers for shot-profile migration. Geophysics 67:883-889

Sava P, Biondi B, Fomel S (2001) Amplitude-preserved common image gathers by wave-equation migration. In: 71st Ann. Internat. Mtg, Soc. of Expl. Geophys., pp 296-299.

Sava PC, Fomel S (2003) Angle-domain common-image gathers by wavefield continuation methods. Geophysics 68:1065-1074

Sava PC, Fomel S (2005) Coordinate-independent angle-gathers for wave equation migration. In: 75th Ann. Internat. Mtg, Soc. Expl. Geophys., Expanded Abstracts. Soc. of Expl. Geophys., pp 2052-2055.

Sava PC, Fomel S (2006) Time-shift imaging condition in seismic migration. Geophysics 71:S209-S217

Slotnick MM (1959) Lessons in Seismic Computing. Society of Exploration Geophysics, Tulsa (Edited by R. A. Geyer).

Soubaras R (2003) Angle gathers for shot-record migration by local harmonic decomposition. In: 73rd Ann. Internat. Mtg., Soc. of Expl. Geophys., pp 889-892.

Stolk C, de Hoop MV (2002) Seismic inverse scattering in the "waveequation" approach. In: CWP-417: Colorado School of Mines.

Stolk CC, Symes WW (2002) Artifacts in Kirchhoff common image gathers. In: 72nd Ann. Internat. Mtg, Soc. Expl. Geophys., Expanded Abstracts. Soc. of Expl. Geophys., pp 1129-1132.

Stolk CC, Symes WW (2004) Kinematic artifacts in prestack depth migration. Geophysics 69:562-575

Stolt RH, Weglein AB (1985) Migration and inversion of seismic data. Geophysics 50:2458-2472

Stork C, Kitchenside P, Yingst D, Albertin U, Kostov C, Wilson B, Watts D, Kapoor J, Brown G (2002) Comparison between angle and offset gathers from wave equation migration and Kirchhoff migration. In: 72nd Ann. Internat. Mtg, Soc. Expl. Geophys., Expanded Abstracts. Soc. of Expl. Geophys., pp 1200-1203.

Vaillant L, Biondi B (2000) Accuracy of common-azimuth migration approximations. In: SEP-103: Stanford Exploration Project, pp $157-168$

Vyas M, Mobley E, Nichols D, Perdomo J (2010) Angle gathers for RTM using extended imaging conditions. In: 80th Ann. Internat. Mtg, Soc. Expl. Geophys., Expanded Abstracts. Soc. of Expl. Geophys., pp 3252-3256.

Xie, XB, Wu RS (2002) Extracting angle domain information from migrated wavefield. In: 72nd Ann. Internat. Mtg, Soc. Expl. Geophys., Expanded Abstracts. Soc. of Expl. Geophys., pp 1360-1363.

Xu S, Chauris H, Lambaré G, Noble M (2001) Common-angle migration: a strategy for imaging complex media. Geophysics 66:1877-1894

Xu S, Zhang Y, Tang B (2010) 3D common image gathers from reverse time migration. In: 80th Ann. Internat. Mtg, Soc. Efxpl. Geophys., Expanded Abstracts. Soc. of Expl. Geophys., pp 3257-3262. 